\title{
Contribuição da Fisioterapia no Tratamento das Alterações Funcionais Decorrentes da Doença Enxerto-Versus-Hospedeiro Crônica Esclerodermoide: uma Revisão de Literatura \\ Physical Therapy Contribution to the Treatment of Functional Changes Caused by Sclerodermoid Chronic Graft Versus Host Disease: a Literature Review Contribución de La Fisioterapia en el Tratamiento de las Alteraciones Funcionales Decurrentes de la Enfermedad Injerto Contra Huésped Crónica Esclerodermoide: una Revisión de la Literatura
}

Daniele Bittencourt Ferreira', Laércio Lima Luz², Inês Echenique Mattos ${ }^{3}$

\section{Resumo}

A doença-enxerto-hospedeiro crônica esclerodermoide é considerada uma das complicaçôes mais severas em pacientes submetidos ao transplante de células-tronco hematopoiéticas, devido à diminuição da amplitude de movimento articular, resultando em alterações funcionais e limitação na realização de atividades de vida diária. Este estudo teve como objetivo discutir a contribuição da fisioterapia no tratamento de pacientes que apresentam redução da amplitude de movimento decorrente da doença-enxerto-hospedeiro crônica esclerodermoide, com base em revisão da literatura. Trata-se de uma revisão sistemática da literatura, através da busca de artigos publicados entre Janeiro de 2000 e Janeiro de 2010, nas bases de dados Medline, Pubmed, SciELO e Lilacs, utilizando os seguintes descritores: doença enxerto-hospedeiro e amplitude de movimento articular. Foram incluídos artigos que abordavam o tratamento fisioterapêutico na doençaenxerto-hospedeiro crônica esclerodermoide. No período selecionado, foram encontrados cinco artigos, mas apenas três preenchiam os critérios de seleção estabelecidos para essa revisão, sendo todos relatos de casos. Os participantes dos estudos apresentavam alteraçóes funcionais semelhantes e se submeteram a tratamento fisioterapêutico, sendo que a maioria evoluiu com melhora funcional e maior independência na realização de atividades de vida diária. Apesar das limitaçôes encontradas nos estudos avaliados, os resultados sugerem que a fisioterapia pode contribuir para a melhora funcional desses pacientes. Desta forma, são necessários outros estudos que utilizem metodologias mais consistentes para que se possa avaliar, de modo mais objetivo, a contribuição da intervençáo fisioterapêutica nos pacientes com alteraçôes funcionais decorrentes da doença-enxerto-hospedeiro crônica esclerodermoide.

Palavras-chave: Doença-Enxerto-Hospedeiro; Terapia por Exercício; Amplitude de Movimento Articular; Literatura de Revisão como Assunto

${ }^{1}$ Especialista em Fisioterapia em Oncologia. Aluna do Programa de Saúde Pública e Meio Ambiente da Escola Nacional de Saúde Pública/ FIOCRUZ.

${ }^{2}$ Especialista em Fisioterapia em Oncologia. Aluno do Programa de Saúde Pública e Meio Ambiente da Escola Nacional de Saúde Públical FIOCRUZ.

${ }^{3}$ Doutora em Ciências da Saúde. Departamento de Epidemiologia da Escola Nacional de Saúde Pública/FIOCRUZ.

Endereço para correspondência: Laércio Lima Luz. Rua Almirante Tamandaré, no 41, apartamento 604. Flamengo - Rio de Janeiro (RJ), Brasil. CEP: 22.210-060.E-mails:danibittfer@hotmail.com; laercioll@hotmail.com; imattos@ensp.fiocruz.br 


\section{INTRODUÇÃO}

O transplante de células-tronco hematopoiéticas é uma modalidade terapêutica utilizada para tratamento de diversas doenças hematológicas, oncológicas, oncohematológicas, metabólicas e imunodeficiências ${ }^{1,2}$. A doença enxerto-versus-hospedeiro (DECH) é considerada a complicação mais severa decorrente do transplante alogênico de células-tronco hematopoiéticas, responsável pela elevada morbimortalidade dos pacientes que a desenvolvem; além de repercutir negativamente na qualidade de vida $^{3}$ e comprometer diversos tecidos, como pele, trato gastrointestinal e fígado ${ }^{4}$. A DECH pode ser classificada em forma aguda, ao se manifestar no período até 100 dias após o transplante; ou crônica, quando sua manifestação ocorre em período superior a 100 dias $^{5}$. Apesar dessa classificação temporal, o mecanismo imunológico que desencadeia a DECH é diferenciado e estudos recentes sugerem que a lesão aguda seria decorrente da ativação de linfócitos $\mathrm{T}$ alorreativos do enxerto; as lesóes crônicas seriam desencadeadas por mecanismos alorreativos e autorreativos ${ }^{6}$.

A DECH é clinicamente significativa, pois atinge cerca de $50 \%$ dos pacientes submetidos ao transplante alogênico, em que $30 \%$ a $50 \%$ desenvolvem a forma crônica e $90 \%$ a $100 \%$ dos casos apresentam alteraçóes cutâneas ${ }^{7}$ geralmente mimetizando doenças autoimunes conhecidas ${ }^{8}$.

Entre as manifestações cutâneas da DECH crônica (DECHc), a esclerodermoide é considerada a forma mais severa de comprometimento devido às alteraçóes funcionais decorrentes de sua evolução ${ }^{1}$. $\mathrm{Na}$ avaliação histológica, observa-se depósito de colágeno tipo I e infiltração perivascular discreta de células mononucleares na derme, atrofia na junçáo derme-epiderme, degeneração de células basais e fibrose da derme. Essas alteraçôes são semelhantes às encontradas na esclerodermia ${ }^{6} \mathrm{e}$ sua progressão manifesta-se através da diminuição da amplitude de movimento e em efeitos secundários, como: perda de força, resistência e capacidade funcional, uma vez que a lesáo que se instala desencadeia um processo de alteraçóes fibróticas da pele, gerando contraturas e envolvendo tecidos contráteis (músculos) e não contráteis (pele, tendóes, cápsulas articulares e fáscias) ${ }^{9}$.

O impacto da limitaçáo funcional repercute negativamente na realização de atividades de vida diária (AVDs), nas relações afetivas e no exercício profissional ${ }^{10}$. A fisioterapia na equipe multidisciplinar que presta assistência ao paciente submetido ao transplante de células-tronco hematopoiéticas encontra-se diretamente relacionada à prevenção de deformidades que poderiam resultar em limitação funcional, através da cinesioterapia motora e utilização de órteses para manutenção ou restauração da amplitude de movimento articular, interferindo positivamente na funcionalidade, na realização das $A V D$ s e na qualidade de vida desses pacientes $^{7,8}$.

Nesse contexto, o objetivo desse artigo é discutir a atuação da fisioterapia em pacientes que apresentaram redução da amplitude de movimento decorrente da $\mathrm{DECHc}$ esclerodermoide, baseada na revisão da literatura existente.

\section{METODOLOGIA}

Foi realizada uma revisão sistemática nas bases de dados Medline, Pubmed, SciELO e Lilacs, através de busca de artigos publicados no período de 1 de janeiro de 2000 a 31 de Janeiro de 2010, com os seguintes descritores: doença-enxerto-hospedeiro e amplitude de movimento articular, nos idiomas português, inglês e espanhol.

Como critério de seleção foram incluídos todos os artigos que abordavam os protocolos de tratamento fisioterapêutico utilizados na DECHc esclerodermoide. Devido à escassez de trabalhos encontrados nas bases de dados pesquisadas, foram incluídos em nossa seleção todos os tipos de estudos.

Os artigos foram ainda categorizados conforme o nível de evidência científica para estudos clínicos do Oxford Centre for Evidence-Based Medicine ${ }^{11}$.

\section{RESULTADOS}

$\mathrm{Na}$ base de dados Medline, foram encontrados apenas dois estudos que preenchiam os critérios estabelecidos nessa revisão.

$\mathrm{Na}$ Pubmed, identificados cinco trabalhos, sendo excluídos dois. Um por abordar apenas o tratamento farmacológico e o outro por tratar somente do tratamento cirúrgico. Entre os três estudos que permaneceram selecionados, dois já haviam sido identificados através do Medline. Nas demais bases de dados pesquisadas, não foram encontrados trabalhos que atendessem aos critérios de seleção.

Foram incluídos nesta revisão, portanto, três artigos, todos eles relatos de casos de pacientes que receberam tratamento fisioterapêutico para alteraçōes funcionais decorrentes da DECHc esclerodermoide (Tabela 1).

Na tabela 1, observam-se estudos realizados nos Estados Unidos, Itália e República da Coreia. Os participantes apresentavam idades variando de 8,6 a 25 anos e o número de casos variou de um a seis. Os pacientes incluídos nesses estudos foram submetidos ao tratamento fisioterapêutico por um período mínimo de quatro semanas ${ }^{1}$ e máximo de um ano ${ }^{7,12}$ e apresentaram alteraçóes funcionais semelhantes, como contraturas em diversas articulações, as quais resultavam em limitações funcionais importantes. Em todos os estudos, o 
Tabela 1. Principais características referentes à atuação fisioterapêutica na DECHc esclerodermoide

\begin{tabular}{|c|c|c|c|c|c|c|c|c|}
\hline $\begin{array}{c}\text { Autor/país/ } \\
\text { ano }\end{array}$ & $\mathbf{N}$ & $\begin{array}{l}\text { Alteração } \\
\text { funcional }\end{array}$ & $\begin{array}{l}\text { Faixa etária } \\
\text { (idade } \\
\text { média) anos }\end{array}$ & $\begin{array}{l}\text { Terapêuticas } \\
\text { aplicadas }\end{array}$ & $\begin{array}{l}\text { Período de } \\
\text { duração do } \\
\text { tratamento }\end{array}$ & $\begin{array}{l}\text { Frequência } \\
\text { ( } n^{0} \text { de } \\
\text { sessões) }\end{array}$ & Resultados & $\begin{array}{l}\text { Nível de } \\
\text { evidência } \\
\text { científica }\end{array}$ \\
\hline $\begin{array}{l}\text { Currie }{ }^{7} \text {, Estados } \\
\text { Unidos, } 2002\end{array}$ & 1 & $\begin{array}{l}\text { Contraturas } \\
\text { severas em } \\
\text { ombros,cotovelos, } \\
\text { punhos, } \\
\text { articulações } \\
\text { interfalangeanas, } \\
\text { quadril e joelhos }\end{array}$ & 14 & $\begin{array}{l}\text { Cinesioterapia } \\
\text { motora } \\
\text { Massoterapia } \\
\text { Parafina }\end{array}$ & 1 ano & $\begin{array}{c}2 \text { vezes por } \\
\text { semana }\end{array}$ & $\begin{array}{l}\text { Independência } \\
\text { para realização } \\
\text { de AVDs }\end{array}$ & 4 \\
\hline $\begin{array}{l}\text { Carenzio'12, } \\
\text { Itália, } 2007\end{array}$ & 6 & $\begin{array}{l}\text { Contraturas } \\
\text { articulares, } \\
\text { anquiloses, } \\
\text { alterações } \\
\text { posturais e } \\
\text { na marcha } \\
\text { e alterações } \\
\text { respiratórias }\end{array}$ & 11,8 & $\begin{array}{l}\text { Cinesioterapia } \\
\text { motora } \\
\text { Massoterapia } \\
\text { Cinesioterapia } \\
\text { respiratória }\end{array}$ & 1 ano & $\begin{array}{l}3 \text { a } 5 \text { vezes } \\
\text { por semana }\end{array}$ & $\begin{array}{l}3 \text { pacientes } \\
\text { obtiveram } \\
\text { melhora } \\
\text { funcional; } \\
2 \text { pacientes } \\
\text { mantiveram- } \\
\text { se estáveis; } \\
1 \text { paciente } \\
\text { apresentou } \\
\text { piora (quadro } \\
\text { clínico) }\end{array}$ & 4 \\
\hline $\begin{array}{l}\text { Choi', } \\
\text { República da } \\
\text { Coreia, } 2009\end{array}$ & 1 & $\begin{array}{l}\text { Múltiplas } \\
\text { contraturas em } \\
\text { articulações, } \\
\text { diminuiç̧ão de } \\
\text { força muscular } \\
\text { nas quatro } \\
\text { extremidades, } \\
\text { alterações } \\
\text { pulmonares } \\
\text { (padrão restritivo) }\end{array}$ & 25 & $\begin{array}{l}\text { Cinesioterapia } \\
\text { motora } \\
\text { Massoterapia } \\
\text { Ultrassom } \\
\text { Parafina }\end{array}$ & 4 semanas & 2 vezes ao dia & $\begin{array}{l}\text { Aumento } \\
\text { discreto da } \\
\text { amplitude de } \\
\text { movimento } \\
\text { articular } \\
\text { em ombros, } \\
\text { cotovelos e } \\
\text { quadris }\end{array}$ & 4 \\
\hline
\end{tabular}

tratamento fisioterapêutico aplicado incluiu massoterapia e cinesioterapia motora; nos resultados finais verificou-se que a maioria dos pacientes apresentou melhora funcional e mais independência para realização de AVDs. Apenas um indivíduo, que evoluiu com piora do quadro clínico, caracterizada pelo agravamento das lesóes de pele, não apresentou melhora funcional ${ }^{12}$. Os trabalhos avaliados são classificados como nível 4 de evidência científica, por se tratarem de relatos e série de casos.

Currie $^{7}$, em seu relato de caso, faz uma descrição completa do quadro de uma paciente de 14 anos de idade que se submeteu ao transplante de células-tronco hematopoiéticas como tratamento para síndrome mielodisplásica e, cerca de um ano e meio depois, apresentou as primeiras manifestaçóes da DECHc esclerodermoide. Com a evolução da doença, a paciente apresentou progressiva perda funcional, sendo necessário um programa de reabilitação mais intenso. A paciente foi atendida por equipe de reabilitação, composta por fisioterapeuta e terapeuta ocupacional, duas vezes por semana, com protocolo de tratamento que incluía o uso de parafina, massoterapia, exercícios, alongamentos e treino para realizaçáo de AVDs. Apesar de os autores não relatarem a utilização de instrumento quantitativo, apresentaram figuras que demonstram, através da mensuração de graus, o aumento da amplitude de movimento em algumas articulações e, após o tratamento, a paciente apresentava independência em AVDs.

Carenzio $^{12}$ avaliou seis crianças (três do sexo masculino e três do feminino), no período de 1999 a 2003, que se submeteram ao transplante de células-tronco hematopoiéticas, não relatando as doenças de base. As avaliações clínicas e funcionais foram realizadas no início da reabilitação e no período de seis a 12 meses de seguimento. As crianças apresentavam DECHc esclerodermoide e importantes alteraçóes funcionais (como 
anquiloses, diminuição da amplitude de movimento de algumas articulaçôes) e respiratórias. O protocolo incluía alongamentos, exercícios de correçáo postural e para recuperar a funcionalidade e a independência para sentar-se, levantar-se e caminhar; massoterapia para redução da contratura muscular e terapia ocupacional, enfatizando movimentos finos realizados pelas máos. Incluía também cinesioterapia respiratória, uma vez que alguns pacientes desenvolveram $\mathrm{DECHc}$ pulmonar. Os pacientes foram acompanhados por um período de 12 meses, com frequência de três a cinco vezes por semana. Ao término do seguimento, três pacientes apresentaram melhora da capacidade funcional e dois mantiveram-se estáveis. Um paciente havia apresentado melhora da funcionalidade, nos primeiros seis meses de tratamento, porém ocorreu piora do quadro clínico com agravamento das lesôes cutâneas, prejudicando o seguimento posterior. É importante observar que esses resultados foram obtidos através da mensuraçáo da amplitude de movimento articular com goniômetro.

Choi ${ }^{1}$ relatou o caso de um paciente de 25 anos com linfoma não-Hodgkin que se submeteu a três transplantes (sendo os dois primeiros autólogos e o terceiro alogênico) e desenvolveu DECHc esclerodermoide. Apresentou diminuição de força muscular em extremidades (grau quatro de força muscular) e múltiplas contraturas em ombros, cotovelos e quadril. Como consequência, em decorrência da redução da amplitude de movimento, o paciente apresentou importante limitação na realização das AVDs. Para avaliação da funcionalidade, os autores mensuraram a amplitude de movimento de ombros, cotovelos e quadril, através de dois instrumentos: Modified Barthel Index (MBI) e Funcional Independence Measure (FMI), tendo o paciente obtido, respectivamente, 96 e 117 pontos, resultados inferiores aos considerados normais. $\mathrm{O}$ programa de reabilitação incluía massoterapia, ultrassom, parafina, alongamento de grupos musculares de grandes articulaçóes, hidroterapia e exercícios para realização de AVDs, duas vezes ao dia, durante quatro semanas, sendo observado aumento discreto da amplitude e movimento articulares. Os autores relatam que, após alta hospitalar, o paciente não se submeteu a qualquer tratamento de reabilitação, apresentando diminuição nas escalas funcionais e na amplitude de movimento.

\section{DISCUSSÃO}

Poucos estudos publicados abordam especificamente a atuação da fisioterapia em indivíduos acometidos pela $\mathrm{DECH}$ cesclerodermoide. Os resultados encontrados nos artigos selecionados por esta revisão sugerem que a abordagem fisioterapêutica poderia ter contribuído para a melhora da funcionalidade, independência na realização de AVDs e aumento da amplitude de movimento articular.
$\mathrm{Kim}^{8}$, em um estudo de caso de um paciente submetido a tratamento cirúrgico em virtude da severidade das contraturas, apontou a importância da atuação fisioterapêutica no tratamento de indivíduos que desenvolvem complicaçóes funcionais decorrentes da $\mathrm{DECHc}$ esclerodermoide, embora não tenha explicitado o protocolo adotado.

Apesar da importância dos estudos de relato ou de série de casos, existem limitaçóes metodológicas importantes no seu desenho, como a ausência de grupo de comparação. A ausência de uma metodologia padronizada que possibilite a reprodutibilidade em estudos subsequentes e o curto período de seguimento são outros pontos que devem ser mencionados. Os estudos publicados sobre a DECHc esclerodermoide não analisam, de forma detalhada, as alterações funcionais que ocorrem com a progressão da doença, dificultando assim a descrição das principais limitações às quais esses indivíduos estão sujeitos.

Os estudos incluídos nesta revisão apresentam algumas limitaçôes específicas que necessitam ser discutidas. $\mathrm{O}$ trabalho de Currie et al. ${ }^{7}$ somente relata a aplicaçáo do FIM (Funcional Independence Measure) no escore relacionado à dependência ao vestir-se e alimentar-se. Carenzio et al. ${ }^{12}$ não relatam o período que transcorreu a partir do diagnóstico da DECHc e o início do tratamento, uma vez que o início precoce de um programa de reabilitação pode minimizar importantes sequelas como anquiloses. No estudo de Choi et al. ${ }^{1}$, o período de tratamento, apesar de intenso, foi muito pequeno, limitando a avaliação dos resultados. Nenhum dos autores informa a duração de cada sessão, o que também pode prejudicar a avaliação da consistência dos achados.

Apesar das limitaçôes, essa revisão contemplou estudos que apresentam as principais alteraçóes da funcionalidade encontradas nos pacientes com DECHc esclerodermoide e que náo são abordadas em estudos clínicos. Os estudos apresentam também os protocolos de tratamento fisioterapêutico que podem ser aplicados em pacientes com essa patologia.

Estudos relacionados a outras patologias ${ }^{13,14} \mathrm{em}$ pacientes com redução da amplitude de movimento articular demonstram a importância da fisioterapia na melhora da funcionalidade e no aumento da independência nas AVDs. A atuação da fisioterapia em pacientes com $\mathrm{DECHc}$ tem como objetivo incrementar a capacidade funcional desses, mesmo que o ganho de amplitude seja parcial, assim como aumentar a independência para a realização de AVDs.

É possível que o período transcorrido entre o diagnóstico de DECHc esclerodermoide e o início do programa de reabilitaçáo possa contribuir para a instalação de sequelas funcionais. Currie et al. ${ }^{7}$, em seu relato de caso, informam que esse intervalo foi de cinco meses e que no momento da avaliação a paciente apresentava limitaçóes funcionais importantes. 
Deve-se ressaltar que os três estudos analisados possuem nível 4 de evidência científica e, portanto, apesar dos resultados positivos apresentados, não se pode afirmar que o benefício da fisioterapia seja incontestável ou que sejam atribuídos exclusivamente ao tratamento fisioterapêutico.

Nesse contexto, torna-se relevante a necessidade de maior produção científica voltada para as alteraçôes funcionais decorrentes da $\mathrm{DECHc}$ esclerodermoide. A realização de estudos que apresentem grupos de comparação, maior número de pacientes e períodos de seguimento mais longos, assim como a utilização de instrumentos de avaliação da funcionalidade padronizados, permitirá uma melhor avaliação da atuação da fisioterapia no tratamento dessas alteraçóes.

\section{CONCLUSÃO}

No período abrangido pela revisão, foram encontrados poucos estudos que abordavam a atuação da fisioterapia no tratamento de pacientes com reduçáo da amplitude de movimento decorrente da DECHc esclerodermoide.

Em virtude do nível de evidência científica dos estudos apresentados nesta revisão, não é possível atribuir os resultados positivos exclusivamente à abordagem fisioterapêutica. Apesar dessas limitaçóes, os resultados sugerem que essa abordagem possa trazer benefícios, como parte integrante do tratamento, contribuindo para a melhora funcional neste grupo de pacientes.

Entretanto, são necessários outros estudos com metodologias mais consistentes para que se possa efetivamente avaliar a contribuição da intervenção fisioterapêutica nos pacientes com alteraçóes funcionais decorrentes da DECHc esclerodermoide.

\section{Declaraçáo de Conflito de Interesses: Nada a declarar.}

\section{REFERÊNCIAS}

1. Choi IS, Jang IS, Han JY, Kim JH, Lee SG. Therapeutic experience on multiple contractures in sclerodermoid chronic graft versus host disease. Support Care Cancer 2009; 17(7): 851-5.

2. Massumoto C. Mizukami S, ALVES A. Transplante de medula óssea. In: Sarmento GJ. Fisioterapia respiratória no paciente crítico: rotinas clínicas. 2 ed. São Paulo: Manole; 2007. p.373-81.
3. Vizoni SL, Lieber SR, Souza CA, Sell AM, VIsentainer JEL. Papel das citocinas na imunopatogênese da doença do enxerto contra o hospedeiro. Revista brasileira de hematologia e hemoterapia 2008; 30 (2):142-52.

4. Häusermann P, Walter RB, Halter J, Biedermann BC, Tichelli A, Itin P, et al. Cutaneous graft-versus- host disease: a guide for the dermatologist. Dermatology 2008; 216 (4): 287-304.

5. Hymes SR, Turner ML, Champlin RE, Couriel DR. Cutaneous manifestation of chronic graft-versus-host disease. Biol Blood Marrow Transplant 2006; 12 (11): 1101-13.

6. Silva MM, Bouzas LFS, Filgueira AL. Manifestações tegumentares da doença enxerto contra hospedeiro em pacientes transplantados de medula óssea. An Bras Dermatol 2005; 80(1):69-80.

7. Currie DM, Ludvigsdottir GK, Diaz CA, Kamani N. Topical treatment of sclerodermoid chronic graft VS. host disease. Am J Phys Med Rehabil 2002; 81(2):143-9.

8. Kim JB, Liakopoulou E, Watson JS. Successful treatment of refractory joint contractures caused by sclerodermatous graft versus host disease. J Plast Reconstr Aesthet Surg 2008; 61 (10):1235-8.

9. Fimiani M, Aloe G De, Cuccia A. Chronic graft versus host disease and skin. J Eur Acad Dermatol Venereol 2003; 17 (5):512-17.

10. Wiskemann J, Huber G. Physical exercise as adjuvant therapy for patients undergoing hematopoietic stem cell transplantation. Bone Marrow Transplant 2008; 41:321-9.

11. Oxford Centre for Evidence-Based Medicine. Levels of Evidence (March 2009). [onlive] [acesso em 19 abr 2010]. Disponível em: URL:http://www.cebm. net $/ ?=1025$.

12. Carenzio G, Gherardi P, Bardoni MT, Zecca M, Bonetti F, Locatelli F, et al. Rehabilitation of chronic graft versus host disease in children - a clinical series. Eura Medicophys 2007; 43(4):445-50.

13. Oliveira MMF, Gurgel MSC, Miranda MS, Okubo MA, Feijó LFA, Souza GA. Efficacy of shoulder exercises on locoregional complications in women undergoing radiotherapy for breast cancer: clinical trial. Rev Bras Fisioter 2009; 13 (2):136-43.

14. Pereira CMA, Vieira EORY, Alcântara PSM. Avaliação de protocolo de fisioterapia aplicado a pacientes mastectomizadas a Madden. Revista brasileira de cancerologia 2005; 51(2):143-8. 


\begin{abstract}
The graft-host disease sclerodermatous is one of the most severe complications in patients who underwent transplantation of hematopoietic stem cells due to a decreased range of articular movement, which results in both functional changes and limitations in performing ordinary chores. This study aimed to discuss the contribution of Physical Therapy to the treatment of patients with reduced range of movement caused by graft-host disease sclerodermatous, based on a literature review. This is a systematic literature review, from articles published between January 2000 and January 2010 in the Medline, PubMed, Lilacs and SciELO databases that were found through the following keywords: Graft versus host disease and range of movement. Articles discussing the physical therapy treatment for graft-host disease sclerodermatous were included. During the selected period, five articles were found, but only three met the selection criteria established for this review, all of which are case reports. The study participants had similar functional changes and underwent physical therapy, most of whom progressed to functional improvement and greater independence in performing ordinary chores. Despite the limitations found in the assessed studies, the results suggest that physical therapy can contribute towards the functional improvement of these patients. Thus, further studies using more consistent methods are needed in order to objectively assess the contribution of physical therapy intervention in patients with functional changes resulting from graft-host disease sclerodermatous.

Key words: Graft vs Host Disease; Exercise Therapy; Range of Motion, Articular; Review Literature as Topic
\end{abstract}

\title{
Resumen
}

La enfermedad injerto contra huésped crónica esclerodermoide es considerada una de las complicaciones más graves en pacientes sometidos a trasplante de células madre hematopoyéticas, debido a la disminución de la amplitud de movimiento articular, ocasionando alteraciones funcionales y limitaciones en la realización de actividades de la vida diaria. Este estudio tuvo como objetivo discutir la contribución de la fisioterapia en el tratamiento de pacientes que presentan reducción de la amplitud de movimiento decurrente de la enfermedad injerto contra huésped crónica esclerodermoide, basado en la revisión de la literatura. Esta es una revisión sistemática de la literatura mediante la búsqueda de artículos publicados entre enero de 2000 y enero de 2010, en las bases de datos Medline, PubMed, SciELO y Lilacs, utilizando las siguientes palabras clave: enfermedad injerto contra huésped y amplitud de movimiento articular. Se incluyeron artículos que abordan el tratamiento de fisioterapia en la enfermedad injerto contra huésped crónica esclerodermoide. En el período seleccionado, fueron encontrados cinco artículos, pero sólo tres cumplieron con los criterios de selección establecidos para esta revisión, y todos eran relatos de casos. Los participantes de los estudios presentaban alteraciones similares funcionales y se sometieran a tratamiento fisioterapéutico, y han avanzado a la mejoría funcional y a una mayor independencia en la realización de actividades de la vida diaria. A pesar de las limitaciones encontradas en los estudios evaluados, los resultados sugieren que la fisioterapia puede contribuir en la mejora funcional de estos pacientes. Así, se necesitan otros estudios que utilicen metodologías más consistentes, para evaluar objetivamente la contribución de la intervención fisioterapéutica en los pacientes con alteraciones funcionales decurrentes de injerto contra huésped crónica esclerodermoide.

Palabras clave: Enfermedad Injerto contra Huésped; Terapia por Ejercicio; Rango del Movimiento Articular; Literatura de Revisión como Asunto 